distinct differences. North TC (NTC): higher cliffs with trails towering over the beach; South TC (STC): lower shoreline cliffs. All injury event data available were reviewed from both sites, including prehospital and media. Medical record and registry data collected include demographics, diagnoses, procedures, outcomes.

Results 159 trauma patients treated at sites were identified. Males comprised $67 \%$, mean age 31.5 years. $48 \%$ occurred between 2014-2016. NTC had more events (54\% vs 41\%), younger patients (29 vs 34 years) and more complex injuries. Mean length of stay was similar (8 vs 7 days) but differences in gender, age and injuries were evident between sites. Activities prior to injury included hiking, hang gliding and intentional jumping into ocean. $47 \%$ with positive alcohol. Fall distance ranged 10-300 feet. 75\% discharged home, 4 hospital deaths, 10 at scene.

Conclusions Coastline cliffs continue to change with erosion and development. Communities continue to fight to preserve natural beauty while ensuring public safety. Incidence increase corresponded with documented surge of social media for gathering and intentional cliff jumping but has since subsided slightly. Visitors, unfamiliar with the environment were common, as well as alcohol use combined with poor judgment; potentially fatal combinations.

Significance and Contributions to Injury and Violence Prevention Science There exists common behaviors, severity and outcomes demonstrating opportunities to develop education and prevention interventions, and still enjoy that sunset.

\section{FARM EQUIPMENT CRASHES ON PUBLIC ROADS: RESULTS FROM A SURVEY OF MIDWEST FARMS}

${ }^{1}$ Matt McFalls, ${ }^{2}$ Corinne Peek-Asa, ${ }^{3}$ Karisa Harland, ${ }^{4}$ Motao Zhu, ${ }^{5}$ Nichole L Morris, ${ }^{1}$ Marizen Ramirez. 'University of Minnesota, School of Public Health; ' University of lowa, Department of Occupational and Environmental Health; ${ }^{3}$ University of lowa, Carver College of Medicine; ${ }^{4}$ Ohio State University, College of Public Health; ${ }^{5}$ University of Minnesota, Roadway Safety Institute

10.1136/injuryprev-2020-savir.135

Statement of Purpose Rural public roads experience higher crash rates than other roadways, with agricultural equipment adding greater risk of injury and fatality. This study investigated farm-level factors and their association with farm equipment crashes on public roads, along with circumstances of these crashes.

Methods/Approac A 2014 survey of nine Midwestern states was analyzed, in which farm operators reported history of farm equipment crashes, details of the crash, and past-year driving of equipment. Chi-square tests were used to compare operator, farm, and vehicle characteristics by crash status. Backward stepwise logistic regression was used to identify predictors of a crash.

Results Of 1403 farms operating equipment on public roads, a weighted $6.4 \%$ reported a crash $(n=100)$. Crops, acreage, vehicle miles, and number of vehicles were initially associated with a crash. Higher odds of crash were observed with second acreage tertile $(\mathrm{OR}=2.98$; 95\% CI: $1.37-6.49)$, third acreage tertile $(\mathrm{OR}=2.72 ; 95 \% \mathrm{CI}: 1.22-6.08)$, and a 10 -vehicle increase $(\mathrm{OR}=1.28 ; 95 \% \mathrm{CI}: 1.02-1.61)$. Crashes occurred most often in June-August (44.3\%). Driver experience was often fewer than five years (83.1\%) and many were paid/ unpaid workers $(48.0 \%)$. Crashes often went unreported to law enforcement (29.0\%).

Conclusions With higher acreage and number of vehicles driven on public roads, farms were more likely to report history of a crash. Many crash characteristics appear to align with findings from previous studies, although differences were observed in seasonality.

Significance and Contributions to Injury and Violence Prevention Science This study suggests further points to explore in the role of large farms and high vehicle usage in farm equipment crashes. These findings provide farm-level context to previous research with motor vehicle crash reports, with the implication that crashes may often go unreported. Limited driver experience may have safety implications for vulnerable high-risk occupations in rural settings.

\section{THE SPATIAL RELATIONSHIP BETWEEN VIOLENT CRIME, BLIGHTED HOUSING, AND EMERGENCY DEPARTMENT UTILIZATION IN HOUSTON, TX}

Alaina Beauchamp. University of Texas Health Science Center at Houston School of Public Health

10.1136/injuryprev-2020-savir.136

Background To identify the relationship between the proximity of crime and blighted housing, and the influence on emergency department (ED) utilization.

Methods/Approach Blighted housing violations and violent physical crime offenses from 2010 were used for spatial analysis of ED utilization. Blight and crime data were taken through open access Houston records, and the ED data was collected in a prior study. Clustering measures (nearest neighbor ratio), frequencies of events within location buffer zones, and spatial autocorrelation calculations were used for analysis.

Results There were 11,741 violent physical crimes, with majority of being aggravated assault (92.34\%). There were 2,969 blight violations, with the largest proportion being 'visual damage is dangerous to life' $(32.06 \%)$. The average number of emergency department visits per month was 15.71 visits per 1000 persons in population $(S D=26.35)$. Blighted housing and violent crime both displayed clustering of the location points $(\mathrm{NNR}=0.23, \mathrm{NNR}=0.31$ respectively $)$. As the number of ED visits per month increases, both the number of blighted properties and crimes increased at a similar rate. Sixty-five percent of all violent physical crimes in Houston occurred within 500 meters of a blighted property $(\mathrm{N}=7595)$. Of all the murders which occurred in Houston in 2010, 83.2\% occurred within approximately five blocks of a blighted house $(\mathrm{N}=208)$.

Conclusions This study provides evidence of the relationship of both factors (e.g. violent crime and blighted housing) on the use of emergency department (ED) services. Future research should look to dissect the reasons for high utilization of emergency services, and the results of criminal actions within Houston.

Significance Although there are many factors that will inevitably influence crime, the blight of the housing in the community may be a large contributor in the perpetuation of violent physical crime. This research supports a link in the blighted housing of the community providing an opportunity for crime and subsequent injury. 